\title{
Inhibiteurs de tyrosine kinase (ITK), un concept et une abréviation en devenir
}

\author{
Patrice Dubreuil
}

> Les leucémies aiguës myéloïdes (LAM) regroupent des hémopathies affectant approximativement 5 personnes sur 100000 et représentent environ $90 \%$ de la totalité des leucémies aiguës de l'adulte [1]. Ce sont des affections clonales, touchant la cellule souche hématopoïétique, caractérisées par une accumulation de cellules blastiques ayant un fort potentiel de prolifération, et dont la différenciation est bloquée à un stade particulier. Les réarrangements cytogénétiques, l'âge et l'historique des syndromes pré-leucémiques sont les déterminants majeurs du pronostic. Bien que la chimiothérapie entraîne une rémission chez 50 à $75 \%$ des patients, seuls $20 \%$ survivront, soulignant la nécessité de développer des traitements complémentaires et/ou plus efficaces. Une de ces nouvelles stratégies, récemment développée dans le cas d'hémopathies, consiste à cibler l'activité tyrosine kinase de récepteurs ou de molécules de signalisation intra-cytoplasmiques par des petits composés chimiques agissant en compétition avec I'ATP. L'utilisation de I'ITK STI571 (Gleevec ${ }^{\circledR}$, Glivec ${ }^{\circledR}$ ), développé par la société Novartis, inhibiteur de la protéine de fusion Bcr-Abl, dans le traitement des leucémies myéloïdes chroniques (LMC) en est un exemple remarquable [2]. Une stratégie analogue pourrait être appliquée dans le cas des LAM en ciblant le récepteur hématopoïétique FLT3. En effet, ce récepteur dont la partie cytoplasmique contient une activité tyrosine kinase de classe 3, au même titre que Kit/SCFR (stem cell factor receptor), Fms/CSFlR (colony-stimulating factor-1 receptor, identique au macrophage colony-stimulating factor receptor) et PDGFR (platelet-derived growth factor receptor), a été décrit à de multiples reprises dans la littérature comme présentant des altérations de type «gain de fonction » dans les LAM. La mutation la plus souvent retrouvée (entre 25 et $30 \%$ des cas selon les études) dans le récepteur FLT3 est une duplication interne en tandem (ITD) d'une séquence palindromique présente dans la région juxta-membranaire du récepteur (codons 591 à 601). Ces duplications peuvent parfois être accompagnées de l'ajout de courtes séquences sans rapport avec FLT3, et sans décalage du cadre de lecture. Plus récemment, un second type de mutation activatrice de FLT3 était identifié (environ $7 \%$ des patients) au niveau de la boucle d'activation du domaine kinase impliquant un acide aspartique en position 835. Ce résidu est homologue à l'Asp ${ }^{816}$ du récepteur Kit affecté dans les mastocytoses systémiques et conférant également aux cellules hématopoïétiques un phénotype de cellule transformée. Les deux types de mutations FLT3 ${ }^{\text {ITD }}$ et FLT3 ${ }^{\mathrm{D} 835}$ conduisent à la dimérisation, l'activation et l'autophosphorylation du récepteur, en l'absence même de la fixation de son ligand (FL, ligand de FLT3). Cette altération se traduit par une activité tyrosine kinase constitutive et une activation des voies classiques de signalisation de FLT3. Ce sont les voies PI3Kinase/AKT, PLC $\gamma$, STAT5 et les voies Ras/Erk Kinases qui sont mises en jeu dans la signalisation de ce récepteur. Plus récemment, on a montré que la reconstitution de souris avec des cellules
Inserm U.119,

Cancérologie expérimentale,

27, boulevard Leï Roure,

13009 Marseille, France.

dubreuil@marseille.inserm.fr

médullaires dans lesquelles les mutations FLT3 ${ }^{\mathrm{ITD}}$ avaient été introduites entraînait le développement d'un syndrome myéloprolifératif sans toutefois de progression vers une leucémie aiguë. Cette altération génétique pouvait donc être considérée comme un événement nécessaire mais non suffisant à l'établissement du processus leucémique [3].

Au cours des deux dernières années, des drogues dont l'activité inhibitrice d'autres récepteurs à tyrosine kinase, VEGFR (vascular endothelial growth factor receptor), PDGFR, Kit ou FGFR (fibroblast growth factor receptor), avait été démontrée, ont été testées pour leur activité inhibitrice de FLT3. Ainsi, I'Herbimycine $A$ ou des Tyrphostines (AG1295 et AG1296), sont certes actives, mais leur toxicité et/ou leur IC50 élevées (concentration de la drogue inhibant $50 \%$ de la prolifération de cellules cibles exprimant FLT3), de l'ordre du micromolaire, s'opposent à toute application thérapeutique $[4,5]$.

Plus récemment, plusieurs publications [6-10] identifiaient des inhibiteurs efficaces de l'activité tyrosine kinase du récepteur sauvage FLT3 activé par son ligand $\mathrm{FL}$, mais également de ses formes constitutivement actives (FLT3 ${ }^{\text {ITD }}$ et $\mathrm{FLT3}^{\mathrm{D} 835}$ ). Ces molécules (CEP-701, PKC412, CT53518, Su5614) développées par quatre compagnies pharmaceutiques différentes (Cephalon, Novartis, Millenium et Sugen) appartiennent à trois familles de molécules différentes: deux sont des dérivés synthétiques de l'alcaloïde staurosporine, une pipérazinyl quinazoline et une indolinone. Leur identifi- 
cation résulte de stratégies de criblage différentes. Le Su5614 fait partie de la large famille d'indolinones développée par la compagnie Sugen contre les kinases des récepteurs VEGFR et Kit et montrant une activité croisée envers FLT3. Le PKC412 et le CEP-701 sont issus d'un criblage biologique, le critère de sélection étant l'inhibition de la prolifération d'une lignée cellulaire ( $\mathrm{Ba} / \mathrm{F} 3$ ) exprimant de façon ectopique un récepteur FLT3 porteur d'une ITD ( $\mathrm{Ba} / \mathrm{F3} \mathrm{FLT3}^{\mathrm{ITD}}$ ), alors que le CT53518 est issu du criblage enzymatique fondé sur l'inhibition de l'activité tyrosine kinase de FLT3. Bien que les deux derniers aient été identifiés par criblage haut débit à partir d'une «bibliothèque » de molécules, seul le CT53518 de Millénium est un nouveau composé. En effet, le PKC412 est un inhibiteur déjà ancien développé par Novartis dès 1989 sous le nom de CGP 41251, connu pour son activité anti-cancéreuse et dirigé contre la protéine kinase C (PKC) [11]; le CEP-701 aussi appelé KT-5555 est quant à lui un analogue de l'indolocarbazole K252a, inhibiteur bien connu des récepteurs de la famille des neurotrophines TRK ( $A, B$ et $C$ ) et dont l'activité anti-tumorale est avérée lorsqu'il y a surexpression de ces oncogènes [12].

Les composés CT53518 et Su5614 ont un spectre d'inhibition relativement restreint, à FLT3 et, à un moindre degré, envers deux autres kinases très proches structuralement, les récepteurs PDGFR $\beta$ et KIT auquel s'ajoute le VEGFR2 pour le Su5614. Les deux autres molécules, en raison de leur homologie structurale avec la staurosporine, ont un spectre d'activité plus $(\rightarrow) \mathrm{m} / \mathrm{s}$ 2001, n० 8-9, p. 952 large. Ainsi, le CEP-701 inhibe efficacement les récepteurs TRK mais également VEGFRl et 2 mais n'exerce aucune inhibition, à faible concentration, de l'activité du PDGFR, de FMS ou de KIT (IC50 $\approx 1 \mu \mathrm{M})$. Le PKC412, outre son activité anti-sérine/thréonine kinase, bloque les RTK de classe 3 (PDGFR $\beta$ et KIT) et de classe 4 (VEGFR2). La spécificité d'action de ces molécules est définie par la valeur de l'IC50 déduite pour chacune de ces kinases par des critères d'inhibition soit biologique, soit enzymatique.

Ainsi, les tests d'inhibition de prolifération ou d'induction d'apoptose réalisés avec les cellules Ba/F3 FLT3 ITD montrent, pour les trois ITK, une IC50 assez voisine aux environs de $10 \mathrm{nM}$ et plus faible pour le Su5614 (200nM). Les données d'inhibition de l'activité enzymatique (mesurée par l'auto-phosphorylation in vitro, la phosphorylation du récepteur ou l'activation de substrats cytoplasmiques du récepteur) semblent indiquer une activité plus importante pour le composé de Cephalon (CEP-701), voisine de son IC50 biologique ( $2 n M)$, que pour le Su 5614 (10 nM) [10], le CT53518 ( $200 \mathrm{nM})$ et le PKC412 ( $\approx 500 \mathrm{nM})$. Toutefois, cette valeur proche du nanomolaire fait craindre une cytotoxicité (et/ou une spécificité d'action large) plus importante du CEP-701, comme le suggèrent les inhibitions de multiples kinases observées à des doses supérieures à $500 \mathrm{nM}$.

Une autre donnée importante, décrite pour le PKC412, le Su5614 et, semble-t-il aussi, pour le CEP-701 (ces données ne sont pas encore publiées) est l'inhibition équivalente induite par ces produits quelle que soit la forme du récepteur qu'il s'agisse de récepteurs normaux ou mutés FLT3 ${ }^{\mathrm{ITD}}$ ou $\mathrm{FLT3}^{\mathrm{D} 835}$. Ce résultat est important si l'on compare avec ceux obtenus pour le STI571 : ce produit bloque de façon très efficace l'activité du récepteur kit sauvage ou muté dans sa partie juxta-membranaire (mutation présente dans les tumeurs gastro-intestinales) $(\rightarrow)$, mais se montre totalement inefficace sur le récepteur $\mathrm{KIT}^{\mathrm{D} 816}$ qui présente une mutation du domaine kinase (analogue du $\mathrm{FLT}^{\mathrm{D} 835}$ ) et caractérise les mastocytoses et certaines LAM [13].

Dans ces quatre études, différents essais biologiques ont confirmé la spécificité de l'activité inhibitrice de ces molécules envers FLT3 et ses mutants constitutifs à potentiel oncogénique. Les composés CEP-701, Su5614 et CT53518 inhibent la prolifération de lignées leucémiques (dans le cas de CEP-701, des cellules pri- maires leucémiques ont été testées) exprimant les mutants « gain de fonction » de FLT3, et entraînent leur apoptose. Ces inhibiteurs ont également permis d'accroître la survie d'animaux porteurs de tumeurs dans deux modèles: (1) CEP-701 et CT53518 se sont révélés efficaces dans un modèle de souris athymiques greffées avec des lignées exprimant FLT3 ${ }^{I T D}$; et (2) dans le modèle précédemment décrit de syndrome myéloprolifératif létal induit après transplantation de cellules médullaires surexprimant FLT3, le traitement par PKC412 et CT3518 entraîne une diminution de la splénomégalie et un rétablissement de l'équilibre entre les différents lignages hématopoïétiques sans toutefois assurer la survie à long terme des animaux. Les données pharmacologiques, et notamment la mesure des concentrations plasmatiques de ces produits après traitement, sont compatibles avec leur IC50 et permettent d'envisager leur utilisation thérapeutique dirigée contre ce récepteur.

En fait, le PKC412, déjà utilisé pour son activité anti-angiogénique dans un essai thérapeutique de phase I chez des patients atteints de tumeurs solides [14], entraîne un très faible effet de myélosuppression pour des doses de 150 à $300 \mathrm{mg} / \mathrm{kg} /$ jour (2 patients sur 32) sans effets secondaires majeurs. Dans cet essai, les concentrations plasmatiques $d u$ produit sont très supérieures à celles qui seraient nécessaires pour inhiber FLT3 [15], un argument qui amène les auteurs à conclure qu'il serait possible d'administrer le produit per os de façon chronique sans redouter une toxicité importante (100 mg/kg/jour).

Une toute récente publication utilisant le CEP701 apporte une autre information intéressante sur la physiopathologie des LAM exprimant des mutations de FLT3 [16]. En effet, l'ajout de cet ITK sur une lignée exprimant FLT3 ${ }^{\text {ITD }}$ permet d'outrepasser le blocage de différenciation en rétablissant le pouvoir inducteur du G-CSF sur cette différenciation. Ce processus de blocage de l'équilibre prolifération-différenciation pourrait donc représenter un mécanisme 
important de la leucémogenèse induite par les mutations affectant FLT3.

Ces différentes publications suggèrent que FLT3 pourrait représenter une bonne cible pour le traitement des LAM et que les ITK apportent un énorme espoir dans la lutte anti-cancéreuse au regard des résultats prometteurs obtenus avec le STI571. Bien sûr, des résistances à ce composé ont été récemment décrites dans la littérature chez des patients traités pour des LMC et ces nouvelles données devront être prises en compte aussi bien sur le plan thérapeutique que sur le plan de la stratégie de recherche et de développement de ces nouveaux médicaments. Ainsi, de nouvelles molécules issues de nouveaux criblages ou optimisées à partir des premières, seront développées. Ces composés pourraient à l'avenir cibler des régions fonctionnelles du domaine kinase différentes du site de liaison à l'ATP, ou des intermédiaires des voies de transduction spécifiquement activées par les oncogènes. Ces nouveaux outils thérapeutiques seront utilisés seuls ou en association avec d'autres ITK ou même en complément des traitements conventionnels. Dans tous les cas, ils apparaissent comme un nouvel outil prometteur dans l'arsenal thérapeutique anti-cancéreux $\diamond$ Tyrosine kinase inhibitors (TKI), both an evolutive concept and an acronym

\section{RÉFÉRENCES}

1. Lowenberg B, Downing JR, Burnett A. Acute myeloid leukemia. $N$ Engl J Med 1999; 341: 1051-62.

2. Druker BJ, Lydon NB. Lessons learned from the development of an abl tyrosine kinase inhibitor for chronic myelogenous leukemia. J Clin Invest 2000; 105: 3-7.

3. Kelly LM, Liu Q, Kutok JL, Williams IR, Boulton $\mathrm{CL}$, Gilliland DG. FLT3 internal tandem duplication mutations associated with human acute myeloid leukemias induce myeloproliferative disease in a murine bone marrow transplant model. Blood 2002; 99: 310-8.

4. Levis M, Tse KF, Smith BD, Garrett $\varepsilon$, Small D. A FLT3 tyrosine kinase inhibitor is selectively cytotoxic to acute myeloid leukemia blasts harboring FLT3 internal tandem duplication mutations. Blood 2001; 98 : 885-7.

5. Tse KF, Allebach J, Levis M, Smith BD, Bohmer FD, Small D. Inhibition of the transforming activity of FLT3 internal tandem duplication mutants from AML patients by a tyrosine kinase inhibitor. Leukemia 2002; 16: 2027-36.
6. Levis M, Allebach J, Tse KF, et al. A FLT3-targeted tyrosine kinase inhibitor is cytotoxic to leukemia cells in vitro and in vivo. Blood 2002; 99: 3885-91.

7. Weisberg $\varepsilon$, Boulton C, Kelly $L M$, et al. Inhibition of mutant FLT3 receptors in leukemia cells by the small molecule tyrosine kinase inhibitor PKC412. Cancer Cell 2002; 1 : 433-43.

8. Kelly $L M, Y u J C$, Boulton $C L$, et al. CT53518, a novel selective FLT3 antagonist for the treatment of acute myelogenous leukemia (AML). Cancer Cell 2002; 1: 421-32.

9. Spiekermann K, Dirschinger RJ, Schwab R, et al. The protein tyrosine kinase inhibitor SU5614 inhibits FLT3 and induces growth arrest and apoptosis in AML-derived cell lines expressing a constitutively activated FLT3. Blood 2002; 24 octobre (online).

10. Yee KW, O'Farrell AM, Smolich BD, et al. SU5416 and SU5614 inhibit kinase activity of wild-type and mutant FLT3 receptor tyrosine kinase. Blood 2002; 100: 2941-9

11. Meyer T, Regenass U, Fabbro D, et al. A derivative of staurosporine (CGP 41251 ) shows selectivity for protein kinase $C$ inhibition and in vitro anti-proliferative as well as in vivo anti-tumor activity. Int J Cancer 1989; 43: 851-6.

12. George DJ, Dionne CA, Jani J, et al. Sustained in vivo regression of dunning $\mathrm{H}$ rat prostate cancers treated with combinations of androgen ablation and Trk tyrosine kinase inhibitors, CEP-751 (KT-6587) or CEP701 (KT-5555). Cancer Res 1999; 59: 2395-401.

13. Zermati, et al. y. Effect of the tyrosine kinase inhibitor STI571 on the kinase activity of wild type and various mutated c-kit receptors found in mast cell neoplasm. Oncogene 2003 (sous presse).

14. Fabbro D, Ruetz $S$, Bodis $S$, et al. PKC412: a protein kinase inhibitor with a broad therapeutic potential. Anticancer Drug Des 2000; 15 : 17-28.

15. Propper DJ, McDonald AC, Man A, et al. Phase I and pharmacokinetic study of PKC412, an inhibitor of protein kinase C. J Clin Oncol 2001; 19: 1485-92.

16. Zheng R, Friedman AD, Small D. Targeted inhibition of FLT3 overcomes the block to myeloid differentiation in 32Dcl3 cells caused by expression of FLT3/ITD mutations. Blood 2002; 100: 4154-61

\section{NOUVELLE}

\section{Un rôle pour le cytosquelette dans les maladies mentales?}

Annie Andrieux, Didier Job

\section{$>$ Les microtubules, \\ des tubes à tout faire. . .}

Les cellules eucaryotes contiennent un réseau d'éléments tubulaires de $25 \mathrm{~nm}$ de diamètre et de longueur variable, de 5 à $50 \mu \mathrm{m}$ selon les types cellulaires. Dans les cellules de mammifères, ces microtubules sont en général polymérisés (nucléés) à partir d'un centrosome. Les réseaux microtubulaires adoptent des configurations variées correspondant à des fonctions également variées [1]. Ainsi, l'organisation de l'appareil de Golgi et des trafics intracellulaires dans les cellules interphasiques, ou la création du fuseau achromatique et la ségrégation des chromosomes durant la mitose
Laboratoire du Cytosquelette, Inserm U.366,

\section{Département réponse et}

dynamique cellulaire,

CEA-Grenoble,

17 , rue des Martyrs, 38054 Grenoble, France. andrieux@dsvsud.cea.fr sont des fonctions dépendantes des microtubules. Les réseaux microtubulaires sont particulièrement abondants dans les neurones. La tubuline représente à elle seule $20 \%$ des protéines neuronales. Les microtubules neuronaux sont nécessaires aux transports dendritiques et axonaux, et la perturbation de 